\title{
Reduction of the effect of aberrations in a joint-transform correlator
}

\author{
Julio Pérez-Tudela, Ignasi Juvelis, Mario Montes-Usategui, Santiago Vallmitjana, and \\ Artur Carnicer
}

\begin{abstract}
We report the study of the influence of optical aberrations in a joint-transform correlator: The wave aberration of the optical system is computed from data obtained by ray tracing. Three situations are explored: We consider the aberration only in the first diffraction stage (generation of power spectrum), then only in the second (transformation of the power spectrum into correlation), and finally in both stages simultaneously. The results show that the quality of the correlation is determined mostly by the aberrations of the first diffraction stage and that we can optimize the setup by moving the cameras along the optical axis to a suitable position. The good agreement between the predicted data and the experimental results shows that the method explains well the behavior of optical diffraction systems when aberrations are taken into account. (C) 2004 Optical Society of America
\end{abstract}

OCIS codes: $\quad 070.2580,080.1010,070.4550,100.4550$.

\section{Introduction}

Optical correlation is an image-recognition method that has been the object of numerous studies for several years. This method is successful in determining the position of a specific pattern inside a complex scene. Optical correlation techniques are based on optical generation of the Fourier transforms (OFTs) of images by means of diffraction. This diffraction can be generated by use of either of two standard setups: the VanderLugt correlator ${ }^{1}$ or the jointtransform correlator ${ }^{2}$ (JTC), which have been widely analyzed and compared. . $^{3,4}$

Usually, when the performance of a correlator is analyzed, its optical systems are considered perfect; i.e., authors do not take into account the optical aberrations introduced by lenses associated with nonparaxial working conditions. In several papers, aberration problems in optical Fourier transformers $^{5-8}$ and even in correlators ${ }^{9,10}$ have been analyzed. However, these papers are somewhat disappointing in that they do not consider the effects of the aberrations on the correlation. In a previous

The authors are with the Departament de Física Aplicada i Òptica, Universitat de Barcelona, Diagonal 647, E08028 Barcelona, Spain. J. Perez-Tudela's e-mail address is julio@optica.ub.es.

Received 27 May 2003; revised manuscript received 8 October 2003; accepted 14 October 2003.

0003-6935/04/040841-09\$15.00/0

(C) 2004 Optical Society of America paper $^{11}$ we presented an analysis of a convergent diffractometer with the object located behind the optical system (the converging architecture allows control of the scale of the Fourier transform by displacing the object along the optical axis). Using a combination of ray and wave optics, we studied the behavior of a VanderLugt correlator that was partially affected by aberrations.

Our aim in this paper is to analyze the aberration effects in the other popular architecture, the JTC. We study how the correlation light distribution is affected by the aberration introduced by the optical systems. In a JTC the correlation is obtained in two Fourier-transform steps: in the first one, a joint power spectrum (JPS) is generated; the second one yields the correlation. We take into account the influence of the aberrations in each OFT and the global effect over the correlation. Using the results obtained in this study, one can deduce the best working conditions for an optical system; moreover, it is possible to compare different optical systems when they are working in their optimal conditions.

The paper is divided into the following sections: in Section 2 the convergent JTC is reviewed, and in Section 3 we present a method of computing wave aberrations that is based on ray tracing and diffraction analysis. Then we take into account the aberrations introduced by two optical systems, namely, a single-meniscus lens and a highly corrected system. Some comparisons between theoretical predictions and experimental results for the first system are pre- 


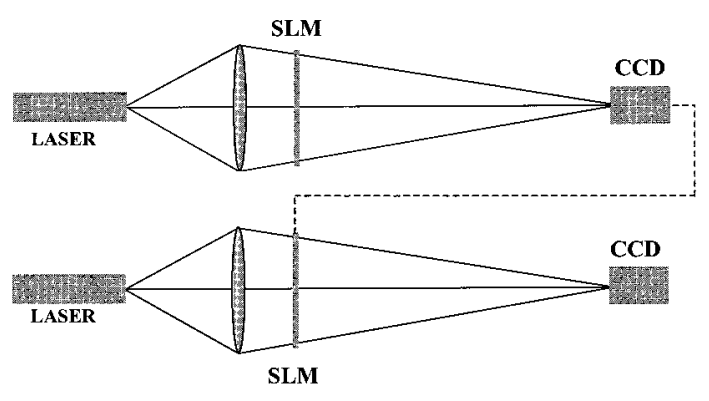

Fig. 1. Convergent JTC.

sented. Finally, we show how to optimize previous results to ensure the best performance for an optical correlator.

\section{Convergent Joint Transform Correlator}

Figure 1 shows a schematic of a convergent JTC: A video camera captures the scene, which is then displayed side by side with the reference image on a spatial light modulator (SLM) placed at the input plane. At the Fourier plane a CCD camera captures the JPS and displays it on a second modulator; finally, a camera placed at the correlation plane captures the result.

We analyzed the arrangement in which each OFT is obtained by means of a converging-beam system (see Ref. 3 for a detailed discussion of the advantages of this system). The Fourier lens is illuminated by a spherical diverging wave front generated by a point source (pinhole), and the OFT is found at the image plane of the source. The optical modulators are placed behind the Fourier lens. The scale of the Fourier transform depends only on the distance between the modulator and the image plane, ${ }^{12}$ so, by tuning this distance, one can easily adapt the size and resolution of the diffraction patterns to those of the CCD camera. Fourier transforms obtained in this way are affected by a multiplicative phase factor, but that is of no importance here because the JTC manipulates only intensity distributions.

The JTC architecture is composed of two convergent diffractometers, each with a light modulator and a CCD camera. The intensity distribution captured by the camera in the first diffraction stage is displayed on the modulator in the second stage, and thus the JTC setup can be implemented by use of a single diffractometer. ${ }^{13}$ The JPS is displayed on the input SLM, and the CCD captures the correlation. If a single diffractometer is used, the OFTs of the input distribution and that of the JPS are performed with the same optical system and, consequently, they will be affected by the same aberration. Nevertheless, we demonstrate that, even in this case, the variation in the distance between the modulator and the CCD permits the reduction of the influence of aberrations.

\section{Method of Wave-Aberration Computation}

We study here the diffraction stage, that is, a converging setup with the diffracting aperture placed

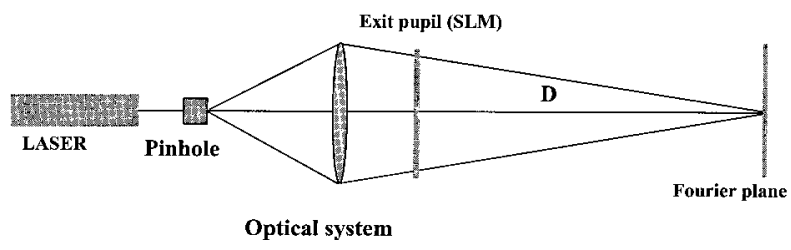

Fig. 2. Sketch of the diffraction system.

behind the lens. Therefore a sketch of the system under analysis is that shown in Fig. 2, composed of the following elements:

- A coherent-light source (laser) of wavelength $\lambda$.

- A point light source (pinhole) that generates the diverging spherical wave front.

- The optical system that generates the converging spherical wave front.

- A SLM, placed behind the optical system, which displays the image to be transformed.

- The Fourier plane, placed a distance $D$ from the modulator, at the image plane of the light source.

The SLM placed behind the lens limits the wave front passing through the system; i.e., it acts as the exit pupil. We compute the complex amplitude distribution at the Fourier plane through the propagation of the wave aberration at the exit pupil. Here, we assume that all diffracting effects are associated with the pupil. Lenses and other stops in the system are assumed to be large enough not to cut the wave front significantly, so propagation of light can be accurately described by geometrical optics from the light source to the SLM (Abbe-Rayleigh theory of image formation ${ }^{14}$ ). Thus, discarding irrelevant constant phase terms for the sake of clarity, we can write the light distribution $U\left(x^{\prime}, y^{\prime}\right)$ at the image plane (our Fourier plane), in a Fresnel approximation, as ${ }^{15}$

$$
\begin{aligned}
U\left(x^{\prime}, y^{\prime}\right)= & \exp \left[-i \epsilon\left(x^{\prime}, y^{\prime}\right)\right] \iint_{\text {Aper }} f(x, y) \\
& \times \exp \left[i 2 \pi\left(\frac{x x^{\prime}+y y^{\prime}}{\lambda R}\right)\right] \mathrm{d} x \mathrm{~d} y,
\end{aligned}
$$

where $\varepsilon\left(x^{\prime}, y^{\prime}\right)$ is a quadratic phase term:

$$
\epsilon\left(x^{\prime}, y^{\prime}\right)=\frac{2 \pi}{\lambda}\left(\frac{x^{\prime 2}+y^{\prime 2}}{2 R}\right)
$$

and $f(x, y)$ is the generalized pupil function:

$$
f(x, y)=t(x, y) \exp \left[i \frac{2 \pi}{\lambda} W(x, y)\right],
$$

where $t(x, y)$ is the pupil transmittance (the image displayed on the modulator) and $W(x, y)$ is the wave aberration, that is, the optical path difference between the wave front generated by the optical system and the reference sphere (see Fig. 3). It is clear from Eqs. (1) and (3) that the quality of the intensity of the 




Fig. 3. Schematic diagram showing an aberrated wave front and its reference sphere.

Fourier transform of $t(x, y)$ depends only on wave aberration $W(x, y)$.

The evaluation of $W(x, y)$ is carried out by means of an exact ray-tracing algorithm, which computes the aberration at different points of the exit pupil according to the relations between wave aberration and ray aberration ${ }^{16}$ :

$$
\frac{\partial W(x, y)}{\partial x}=-\frac{X}{R-W}, \quad \frac{\partial W(x, y)}{\partial y}=-\frac{Y}{R-W},
$$

where $X$ and $Y$ are the components of the ray aberration (difference between the actual impact point and that predicted by paraxial optics) and $R$ is the radius of the reference sphere. The ray-tracing algorithm thus evaluates the wave aberration at discrete points, those that result from the intersection of the traced rays with the exit pupil plane. The computation of $W(x, y)$ on the entire pupil is carried out by interpolation of the discrete values with an aberration polynomial of the type ${ }^{17}$

$$
\begin{aligned}
W(x, y)= & A_{1}\left(x^{2}+y^{2}\right)+A_{2}\left(x^{2}+y^{2}\right)^{2} \\
& +A_{3}\left(x^{2}+y^{2}\right)^{3}+\ldots,
\end{aligned}
$$

where only terms that express radial symmetry have been included in the expansion because the diffraction stage works with a point source located at the axis of the optical system. If we neglect wave aberration $W$ (a few wavelengths) in front of $R$ (several centimeters in our case), Eqs. (4), after differentiation of Eq. (5) with respect to $x$ and $y$, become

$$
\begin{aligned}
-\frac{X}{R}= & 2 A_{1} x+4 A_{2} x\left(x^{2}+y^{2}\right)+6 A_{3} x\left(x^{2}+y^{2}\right)^{2} \\
& +\ldots, \\
-\frac{Y}{R}= & 2 A_{1} y+4 A_{2} y\left(x^{2}+y^{2}\right)+6 A_{3} y\left(x^{2}+y^{2}\right)^{2} \\
& +\ldots
\end{aligned}
$$

\begin{tabular}{|c|c|c|}
\hline \multirow[b]{2}{*}{ Radii for Lens $1(\mathrm{~mm})^{a}$} & \multicolumn{2}{|r|}{ Medium } \\
\hline & Thickness & $\begin{array}{l}\text { Index (Abbe } \\
\text { Number) }\end{array}$ \\
\hline$R_{1}=-128.26 \quad R_{2}=-59.37$ & $5.44 \mathrm{~mm}$ & $n_{1}=1.523\left(v_{d}=59\right)$ \\
\hline
\end{tabular}

For each traced ray we have two linear equations with $k$ unknowns, $A_{1}, A_{2}, \ldots, A_{k}$, the coefficients of
Table 1. Meniscus Lens Data

${ }^{a}$ Focal length, $206.5 \mathrm{~mm}$.

the aberration polynomial. The remaining information (ray aberrations, ray intercepts at the exit pupil) are all available through the ray-tracing algorithm. We typically compute several rays $(n>k)$ and obtain an overdetermined set of linear equations, which is then solved by minimization of the quadratic error between the two sides of Eq. (6). Once the coefficients of the aberration polynomial have been found, the generalized pupil function is computed according to Eq. (3). Finally, the distribution of light at the Fourier plane is obtained by transformation of the pupil function by means of a discrete Fouriertransform algorithm.

\section{Optical Systems and Experimental Configuration}

To analyze the behavior of the correlator as a function of the degree of aberration, we have taken two different optical systems with the same focal length into consideration. The first one consists of a singlemeniscus lens and the second one is a more-complex system (Meopta objective), with four lenses and better optical quality. See Tables 1 and 2 and Fig. 4 for details.

The target used for detection is the image of a fish. The scene is the same fish displayed at eight positions on the input modulator to produce input images with variable amounts of aberration and to permit the loss of spatial invariance in the correlator to be investigated. The target is placed at the center. Figure 5 shows the centered target and the eight scenes superimposed.

Note that, as we are dealing with aberrated optical systems, we do not have an exact Fourier transform at the image plane of the light source but only an approximation. This means that we are not required to use that plane as the Fourier plane of the correlator. Defocusing may compensate to some extent for the aberrations of the optical system; therefore a better approximation of the Fourier transform may be found at a different position along the optical axis. Such has indeed been our experience, as we show in the following results. Figure 6 shows plots of the wave-aberration polynomial for different positions of the Fourier plane as a function of radial coordinate $r$ :

$$
r=\left(x^{2}+y^{2}\right)^{1 / 2} .
$$

The eight positions of the scene are indicated by vertical lines. Figure 6(a) (meniscus lens) shows six aberration polynomials that correspond to the paraxial plane and six positions of the image plane. Fig- 


\begin{tabular}{|c|c|c|c|c|}
\hline \multirow[b]{3}{*}{$\begin{array}{c}\text { Lens } 1 \\
\text { Air }\end{array}$} & & & \multicolumn{2}{|r|}{ Media } \\
\hline & \multicolumn{2}{|c|}{ Radii (mm) } & Thickness (mm) & Index and (Abbe Number) \\
\hline & $R_{1}=283.19$ & $R_{2}=-389.42$ & $\begin{array}{c}17.9 \\
1.38\end{array}$ & $n_{1}=1.61375\left(v_{d}=59\right)$ \\
\hline $\begin{array}{c}\text { Lens } 2 \\
\text { Air }\end{array}$ & $R_{3}=100.93$ & $R_{4}=302.47$ & $\begin{array}{l}30.96 \\
11.02\end{array}$ & $n_{2}=1.620410\left(v_{d}=62\right)$ \\
\hline $\begin{array}{c}\text { Lens } 3 \\
\text { Air }\end{array}$ & $R_{5}=-169.45$ & $R_{6}=80.59$ & $\begin{array}{l}10.32 \\
24.78\end{array}$ & $n_{3}=1.717360\left(v_{d}=62\right)$ \\
\hline Lens 4 & $R_{7}=476.44$ & $R_{8}=-86.32$ & 24.78 & $n_{3}=1.620410\left(v_{d}=62\right)$ \\
\hline
\end{tabular}

${ }^{a}$ Focal length, $206.5 \mathrm{~mm}$.

ure 6(b) (meopta objective) shows five aberration polynomials at different planes that correspond to the paraxial distance and four other positions. Distances are given with respect to the paraxial image position of the point source: The paraxial plane is $d=0$; planes farther away from the lens than the paraxial plane take positive values, and closer planes take negative values.

To verify the validity of the method we designed an experimental on-axis JTC. Our aim in using this setup was to reproduce the simulated working conditions as closely as possible, so the meniscus lens described in Table 1 was used as the diffractive system. The light source was a He-Ne laser $(\lambda=632.8 \mathrm{~nm})$ located at the object plane [Fig. 4(a)], and a CCD camera was placed at the image plane. The image to be transformed, which included the fish to be detected and the centered reference fish, was located at the exit pupil and was obtained in the form of a photographic negative to prevent the appearance of any undesirable effects from electro-optical elements.

\section{Effects of Aberrations on the Correlation Results}

The method allows us to isolate the effect of aberrations in each of the two Fourier-transform steps in-

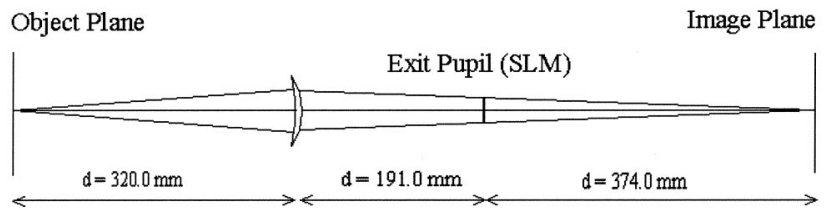

(a)

Object Plane

Image Plane



(b)

Fig. 4. (a) meniscus lens, (b) meopta objective. volved in a JTC and to study how they separately affect the final correlation.

\section{A. Effects of Aberrations in the First Diffraction Stage}

To evaluate how the aberration of the first diffraction stage affects the final correlation, we captured the JPS with a CCD camera placed at the image plane and then transformed it by means of a fast-Fouriertransform algorithm. This procedure isolates the effect of the aberrations that arise in the first diffraction stage, inasmuch as the second Fourier transform is digital and thus without aberrations. The values of the correlation peak heights are compared to those that result from the aberration-free case.

Figure 7 shows the relative height of correlation peaks, that is, the ratio between the correlation value for which aberrations are considered only in the first diffraction stage and that of an ideal correlator with no aberrations. Different scene positions and capture planes for the JPS are represented in the figure.

Figure 7(a) corresponds to the simulated results obtained, and Fig. 7(b) shows the experimental results. From an analysis of these figures it can be concluded that the peak heights in the paraxial plane exhibit an unstable behavior; that is, the results show

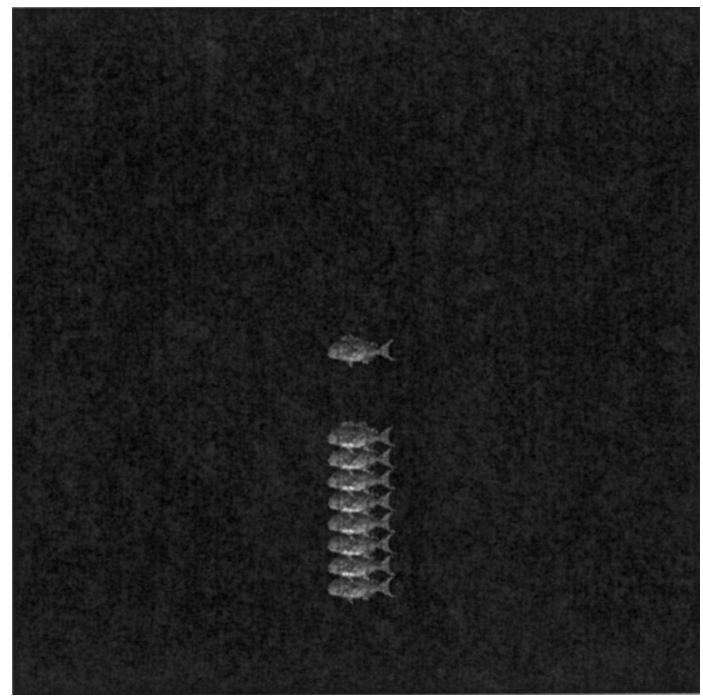

Fig. 5. Centered target and eight scenes superimposed. 


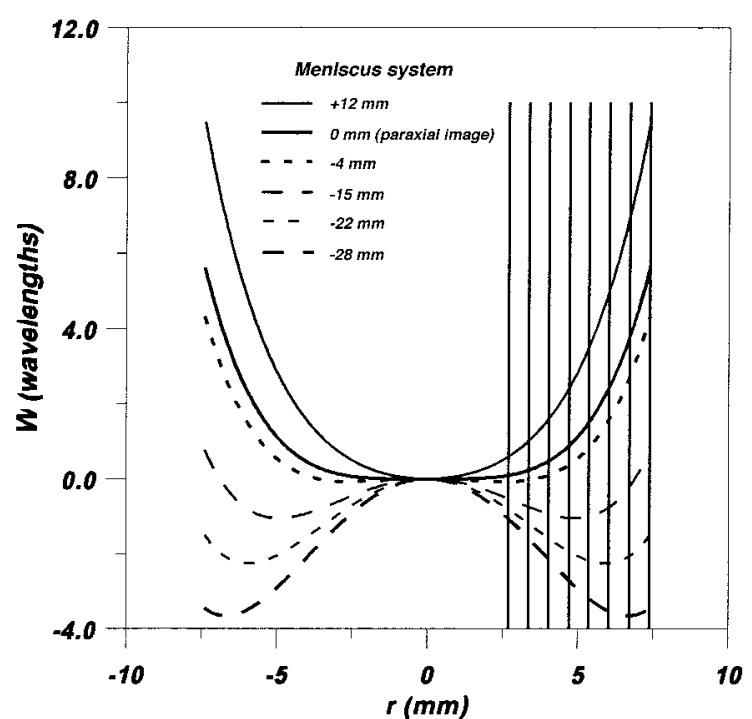

(a)

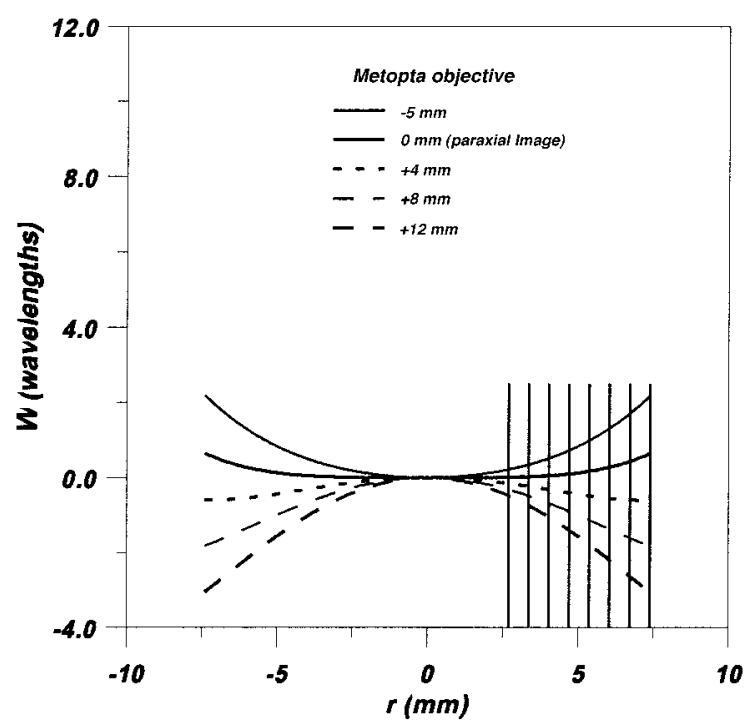

(b)

Fig. 6. Aberration polynomial for several image planes (a) for a meniscus system and (b) for a meopta objective. The eight positions for the scene are indicated by vertical lines.

a strong spatial variance. The peak values decrease quickly when the target is far off the center of the scene. If we move away from the paraxial plane $(d=+12 \mathrm{~mm})$ the behavior is even worse. Nevertheless, if we come closer to the system, the height of the peaks becomes more stable. The best results are obtained for $d=-15 \mathrm{~mm}$, where the peak values are stable for targets in a fairly large neighborhood near the center of the scene.

One can obtain stable behavior of the correlation peak values by choosing a suitable plane, closer to the lens than the paraxial plane, with which to capture the OFT. However, for targets located far from the center of the scene, total invariance of the height of the peaks is not possible.

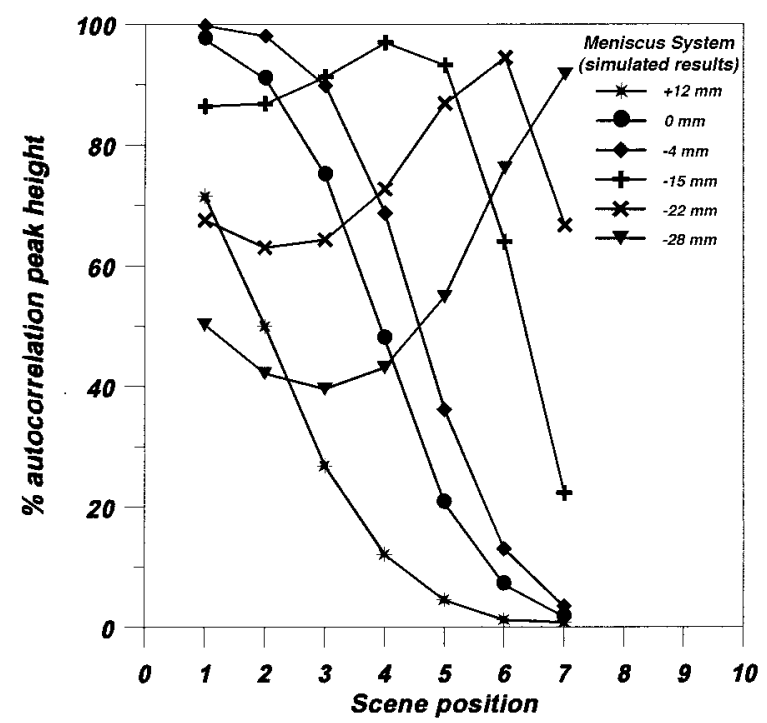

(a)

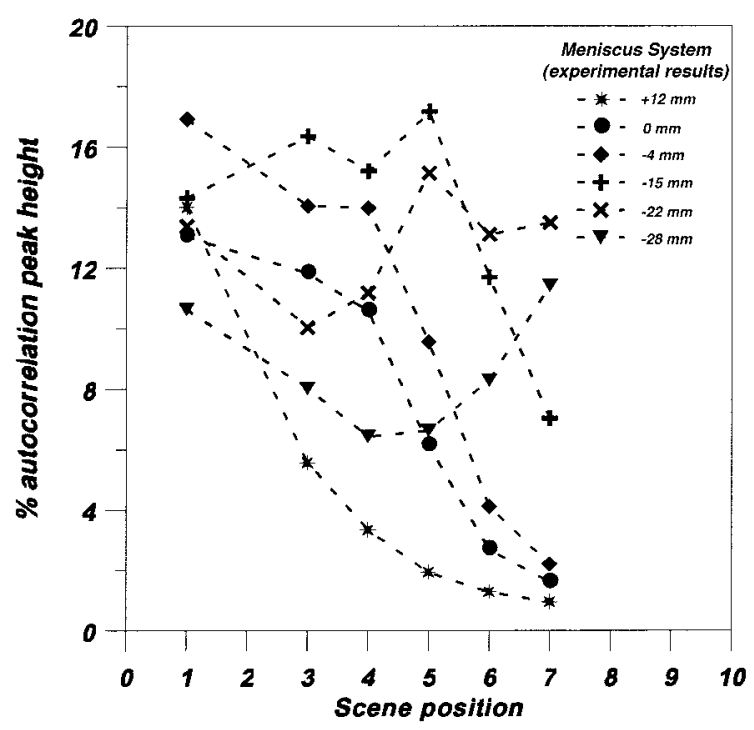

(b)

Fig. 7. Relative height correlation peaks. Aberration in only first OFT. meniscus lens: (a) simulated results, (b) experimental results.

\section{B. Effects of Aberration in the First and Second Transforms}

In this section we describe aberration in both the first and the second transforms. In this case we have two transforms and consequently two possible positions of the image plane. Nevertheless, we have considered the position of the Fourier plane to be the same in both steps. This corresponds to the practical case in which a single optical setup is used twice sequentially ${ }^{13}$ (as opposed to two different stages in series).

The experimental process developed in this situation is different from that described in Subsection 5.A in that the second transform is not digital. The JPS captured by the CCD camera is printed 


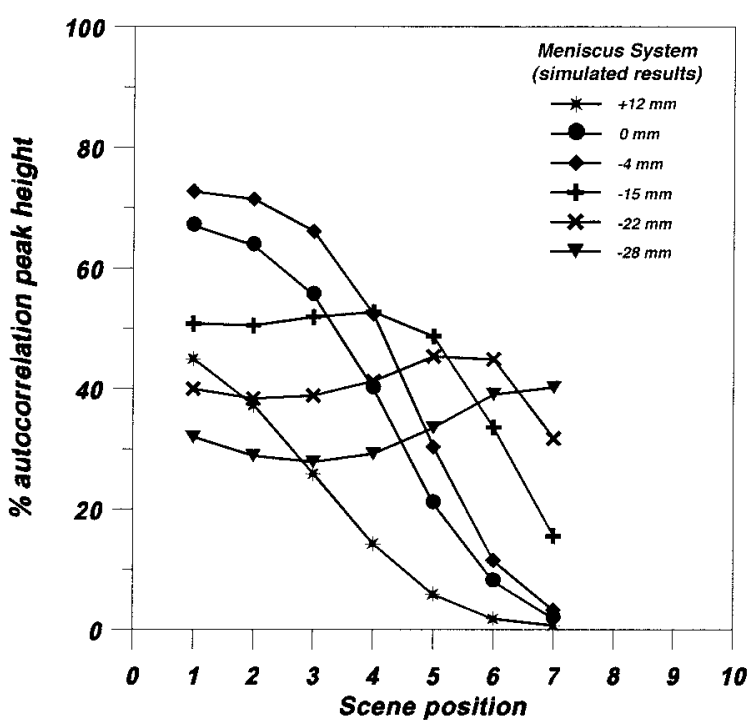

(a)

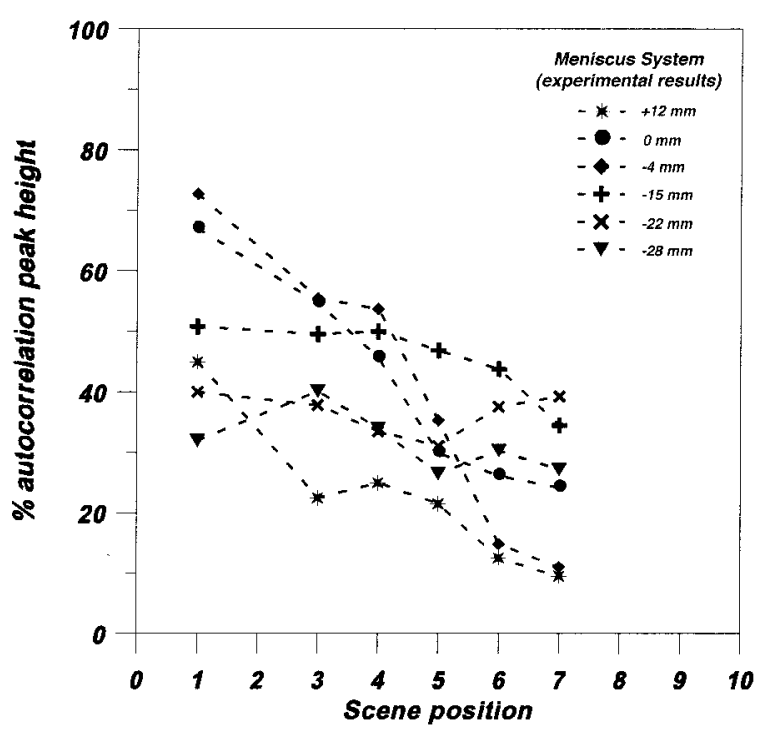

(b)

Fig. 8. Relative height correlation peaks. Aberration in first and second OFTs. Meniscus lens: (a) simulated results, (b) experimental results.

and transformed into a photographic negative. This image is located at the exit pupil of the JTC, and then the correlation image is obtained at the image plane. Finally, we analyze the images to obtain the relative values of the correlation peak heights as before.

In Figs. 8(a) and 8(b), respectively, simulated and experimental results are presented. It can be observed that the curves have a behavior similar to those shown Figs. 7(a) and 7(b) but with a noticeable decrease in their values. The curve that corresponds to the paraxial plane $(d=0)$ shows the largest variation, and, in contrast, that which corresponds to $-15 \mathrm{~mm}$ is almost constant. This invariance, as in the preceding case, can be attributed

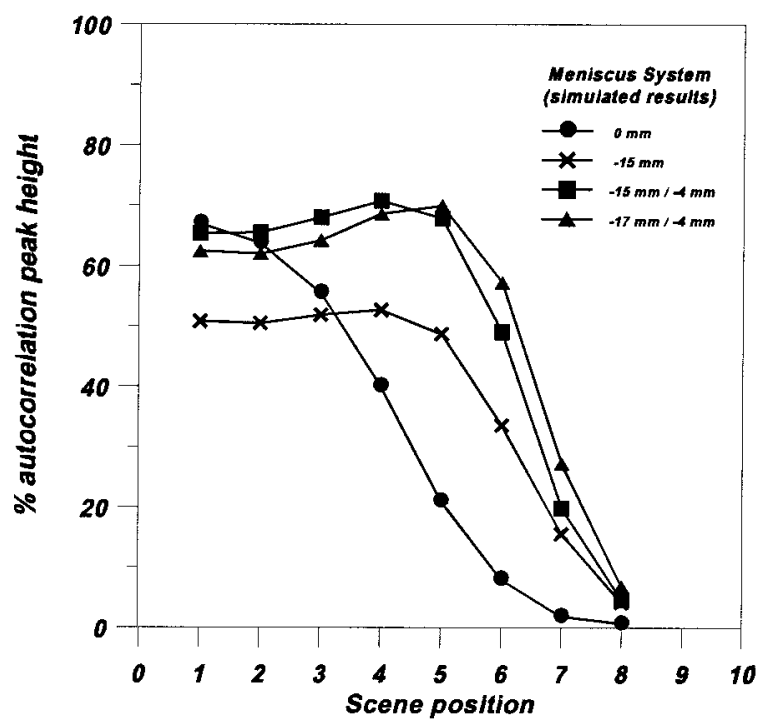

Fig. 9. Relative height correlation peaks. Meniscus lens. Different image plane positions in first and second transforms.

to the aberrations in the first transform. The effect of aberrations in the second transform is a global decrease of the curves: the curve that corresponds to the paraxial plane goes down by roughly $30 \%$ (from approximately $100 \%$ to $70 \%$ ), and the one that corresponds to plane at $-15 \mathrm{~mm}$ goes from some $90 \%$ to $50 \%$.

Then, considering all the aberrations and the various capture positions for the CCD, it is possible to find a plane in which the spatial invariance is preserved. However, the values of correlation maxima decrease because of the effect of aberrations in the second OFT. It is worth mentioning that this is a general pattern, whether or not the first transform is also affected by or free from aberrations. That is why we do not include a separate study of the aberrations in the second diffraction stage only. The peaks behave in the same way as in the aberrationfree case, except that they are lower.

\section{Optimization of the Position of the Image Plane}

In Subsection 5.B we analyzed the effect of the aberrations on the height of the correlation maxima when the detector at the Fourier plane is placed at the same position in both transforms. One also can move the detector to various positions and acquire the JPS and the correlation at different distances from the paraxial plane. This corresponds to the experimental situation of operating with two diffracting setups or with a single processor and moving the position between the two transforms.

From Fig. 7(a) we have seen that the best conditions for correlation appear when the power spectrum is obtained in a plane located at $-15 \mathrm{~mm}$ from the paraxial spectrum. We kept this position fixed and optimized the capture position of the second transform. The results obtained are those shown in Fig. 9. Here the first OFT is captured at -15 or $-17 \mathrm{~mm}$ and the second OTF is acquired at a different plane. 


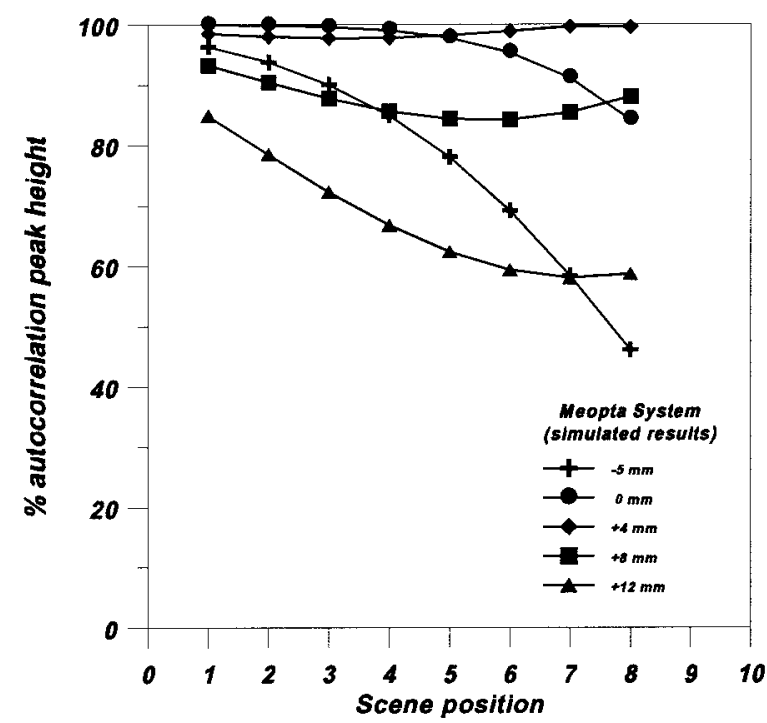

(a)

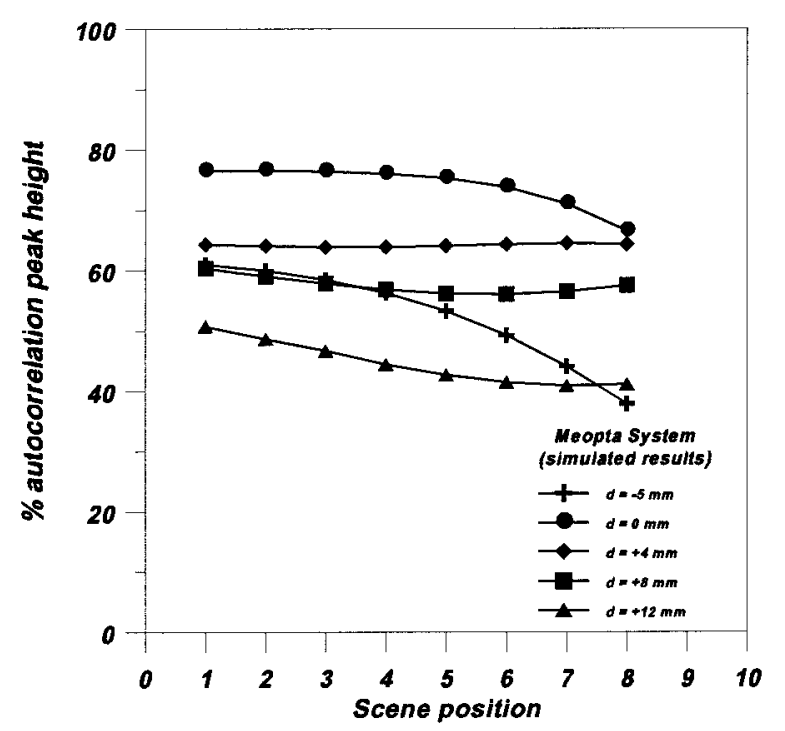

(b)

Fig. 10. Relative height correlation peaks. Meopta objective (simulated results): (a) aberration in first OFT only, (b) aberration in first and second OFTs.

As can be seen, by choice of a suitable combination of the positions of the acquisition planes it is possible to obtain higher values of correlation peaks as well as a larger degree of spatial invariance [curves $d=0$ and $d=-15$ in Fig. 7(a) are also included here for comparison]. The best combination of planes corresponds to a first transform at $d=-15 \mathrm{~mm}$ and to the second one at $d=-4 \mathrm{~mm}$.

\section{Highly-Corrected Optical System}

The experimental verification presented above allows us to ensure that the method developed is a good model of the diffractive behavior of optical systems used in the correlation process. The next step was to apply the method to more-sophisticated systems and

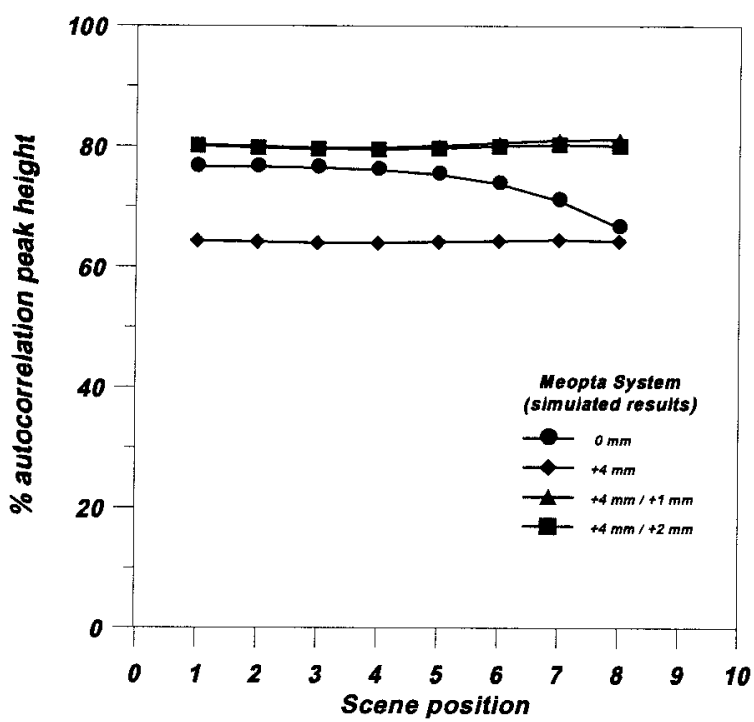

Fig. 11. Relative height correlation peaks. Meopta objective. Different image plane positions in first and second transforms.

to determine whether the effects observed in the correlation, when one is working with a single-meniscus lens, still appear when a system with a better optical quality is used. To take the second step we reproduced the whole process explained in Section 4 as we worked with the meopta objective described in Table 2 and Fig. 4(b). The results obtained were almost the same as those obtained for the meniscus lens, as shown in Figs. 10 and 11.

For Fig. 10(a) we considered aberration only in the first diffraction stage. It can be observed that, at $d=0 \mathrm{~mm}$, the heights of the peaks vary with target position; at $d=-5 \mathrm{~mm}$ this effect is still more evident. When the OFT is evaluated at $d=+4,+8$, $+12 \mathrm{~mm}$, however, the peak values are more stable. As can be seen, the best behavior is obtained when the plane is placed at $d=+4 \mathrm{~mm}$. As the meopta objective is a better optical system than the singlemeniscus lens, the more suitable plane is found closer to the paraxial plane, and global stable behavior can be achieved.

If the aberrations in the two diffraction stages are considered [Fig. 10(b)], we can also attain invariance of the correlation values by changing the position of the image plane (at $d=+4 \mathrm{~mm}$ ) in the first Fourier transform. However, the effect of the aberrations in the second transform decreases the value of the correlation peak heights from $100 \%$ to some $65 \%$.

Finally, as can be seen from Fig. 11, it is also possible to find a combination of acquisition planes that increases the values of correlation peaks without losing the spatial invariance. In this case the best combination corresponds to a first transform captured at $d=4 \mathrm{~mm}$ and the second one at $d=1,2 \mathrm{~mm}$. Based on these results, it can be concluded that the behavior of a well-corrected optical system does not differ significantly from that observed for the single-meniscus lens. Nevertheless, as presumed, the effects of the 
aberrations of the diffractive system in the final correlation were not so marked as in the previous case.

\section{Conclusions}

We have analyzed the effect of the aberrations associated with optical systems in a joint-transform correlator with a converging diffractometer architecture by computing the wave aberration by exact ray tracing. As the JTC has two identical Fouriertransform optical setups, we studied the effect of the aberrations in each one separately by both simulation and experiment.

We analyzed the global effect over the height of the correlation maxima. One of the problems that we found is that the presence of aberrations produces a space-variant output. To study this effect we used scenes with the target placed at eight different positions with respect to the reference image.

We obtained the first set of results by considering the influence of the aberrations only in the first optical Fourier transform (joint power spectrum) and considering the second OFT perfect. In these conditions the main relevant conclusions are that

- If we place the first Fourier plane (corresponding to the power spectrum) at its theoretical paraxial position, the final correlation is strongly space variant, that is, the height of the correlation peak depends on the position of the scene image.

- If we slightly move the plane of the device that captures the power spectrum from the paraxial position, the variations of the correlation output can be decreased.

- It is possible to find a Fourier plane position where aberrations have almost no effect and the correlation peak height is nearly invariant within a fairly large neighborhood. For the single-lens system (meniscus lens), the peak value invariance is achieved only for five of the eight tested target positions. Better performance is obtained (full invariance for those eight positions), however, when the meopta objective is used.

In the second step we considered the influence of the aberrations in both OFTs. First we considered that the position of the detector is the same in each transform. This situation corresponds to the experimental setup in which the two transforms are captured sequentially in the same optical bench. In this situation we conclude that

- The behavior of the correlation is analogous to that in the previous situation: It is possible to find a position for locating acquisition planes where the space-variant effect related to paraxial distance decreases. The heights of all the correlation peaks remain almost constant but lower: approximately $50 \%$ (meniscus lens) and 65\% (meopta objective) of the heights that correspond to theoretical values (aberration free).
The simulated behavior of one of the optical systems (meniscus lens) has been corroborated by means of an experimental setup that reproduced the same two configurations, namely, when the aberration appears only in the first diffraction stage and when both transforms are taken into account. The good agreement between experimental and numerical results proves the value of the method in predicting the influence of optical aberrations in the final correlation for real systems.

Finally, we combined different displaced positions of the OFT acquisition planes, with the following conclusion:

- Among all possible combinations it is possible to find one optimal trade-off position at which the correlation peaks are space invariant despite the presence of aberrations. With this configuration, all peaks are $\sim 70 \%$ (meniscus lens) or $80 \%$ (meopta objective) of the value without aberration.

In summary, the aberrations of Fouriertransforming optical systems used in a JTC strongly affect the correlation results. The aberrations on the first transform induce space variance in the final correlation, and the aberrations on the second transform produce a global decrease in the heights of the correlation peaks. The experiments performed corroborate this behavior. By selection of a suitable combination of positions of the two acquisition planes it is possible to compensate for both effects and to find an optimal configuration in which the correlation peaks are sharp and almost invariant.

This research has been supported in part by the Spanish R and D agency (Comisión Interministerial de Ciencia y Tecnología) under project DPI20013365 .

\section{References}

1. A. VanderLugt, "Signal detection by complex spatial filtering," IEEE Trans. Inf. Theory IT-10, 139-145 (1964).

2. C. S. Weaver and J. W. Goodman, "A technique for optically convolving two functions," Appl. Opt. 5, 139-145 (1966).

3. D. Joyeux and S. Lowenthal, "Optical Fourier transform: what is the optimal setup?" Appl. Opt. 21, 4368-4372 (1982).

4. X. J. Lu, F. T. S. Yu, and D. A. Gregory, "Comparison of VanderLugt and joint transform correlator,” Appl. Phys. B 51, 153-164 (1990).

5. K. Von Bieren, "Lens design for optical Fourier transform systems," Appl. Opt. 10, 2739-2742 (1971).

6. S. Bosch, S. Vallmitjana, I. Juvells, and J. R. de F. Moneo, "Scale-tunable diffractometer for spatial light modulators, a design procedure," in Lens and Optical Systems Design, H. Zuegge, ed., Proc. SPIE 1780, 592-601 (1992).

7. D. Casasent and T. Luu, "Phase error model for simple Fourier transform lenses," Appl. Opt. 17, 1701-1708 (1978).

8. D. Casasent and T. Luu, "Performance measurement techniques for simple Fourier transform lenses," Appl. Opt. 17, 2973-2980 (1978).

9. D. J. Lanteigue, "Optical aberrations of correlators," in Digital 
and Optical Shape Representation and Pattern Recognition, R. D. Juday, ed., Proc. SPIE 938, 36-39 (1988).

10. S. A. Comastri, "Joint transform correlator: expansion of the aberration function for a compact design," Pure Appl. Opt. 4, 753-770 (1995).

11. J. Pérez-Tudela, M. Montes-Usategui, I. Juvells, and S. Vallmitjana, "Analysis of the influence of aberrated convergent Fourier-transform setups in optical correlation," Opt. Commun. 184, 345-355 (2000).

12. S. Vallmitjana, J. Pérez-Tudela, M. Montes-Usategui, I. Juvells, and A. Carnicer, "Influence of the aberrations of optical Fourier-transform systems in a joint transform correlator," in Wave Optics and VSLI Photonic Devices for Information Pro- cessing, P. Ambs and F. R. Beyette, Jr., eds., Proc. SPIE 4435, 42-49 (2001).

13. B. Javidi and J. L. Horner, "Single spatial light modulator joint transform correlator," Appl. Opt. 28, 1027-1032 (1989).

14. J. W. Goodman, Introduction to Fourier Optics (McGraw-Hill, New York, 1968).

15. H. H. Hopkins and M. J. Yzuel, "The computation of diffraction patterns in the presence of aberrations," Opt. Acta 17, 157-182 (1970).

16. J. L. Rayces, "Exact relation between wave aberration and ray aberration," Opt. Acta 11, 85-88 (1964).

17. W. T. Welford, Aberrations of Optical Systems (Adam Hilger, Boston, Mass., 1986), p. 1981. 